\title{
Carrillo Huerta, Mario Miguel, Estudios regionales en M éxico. Selección de teoría y evidencia empírica: desarrollo regional, Puebla, Universidad de Puebla, 2002
}

\section{G ustavo G arza*}

El objetivo principal del libro es "ofrecer una selección de estudios de desarrollo de diferentes regiones mexicanas que el autor ha realizado durante un periodo más o menos largo (1980-2002)" (p. 2). ${ }^{1}$ Se pretende posibilitar que la información estadística incluida en dichos artículos, así como la metodología que los sustenta "sirvan de apoyo en los cursos de desarrollo regional que ofrezco en programas formales de educación superior, a los niveles de licenciatura y posgrado" (p. 2). Se advierte claramente que se trata de un libro de texto y únicamente en esos términos se debe analizar.

El trabajo se estructura en 10 capítulos que se agrupan en cuatro partes: la parte I es la introducción general y fue subdividida en tres capítulos: el primero es teórico y presenta los conceptos fundamentales de las teorías del desarrollo regional, tanto en los aspectos de delimitación de las unidades regionales y su relación con las estructuras socioeconómicas, como en la problemática relativa a los estudios regionales de caso. El segundo describe en forma sistemática la trayectoria de las desigualdades regionales en México hasta la última década del siglo pasado y el papel de la intervención del Estado en relación con la inversión pública federal. El tercero detalla las estrategias regionales del gobierno federal por medio de las cuales ha intentado incidir en la evolución económica de las diferentes regiones del país con el propósito de reducir las desigualdades regionales.

La parte II, Estudios en regiones, consta de dos capítulos, ambos centrados en la región del norte de México, una de las zonas en que ha sido más fuerte el impacto del experimento neoliberal de apertura del país al mercado internacional; así, los capítulos 4 y 5 se refieren a las importantes cuestiones que determinan la relación de la zona con Estados Unidos, y al impacto de la dinámica industria maquiladora.

* Profesor-investigador del Centro de Estudios Demográficos y de Desarrollo Urbano de El Colegio de México. Correo electronico: ggarza@colmex.mx.

${ }^{1}$ Para las citas del libro que se esta reseñando sólo se presenta el número de la página. 
La parte III está constituida por tres capítulos (6, 7 y 8) que tratan sobre el impacto de los programas regionales de empleo en Puebla en 1985; la industrialización en Baja California; las secuelas ecológicas del crecimiento económico de Chiapas. Finalmente, en el capítulo 9 de la parte IV se presentan las experiencias de promoción del desarrollo de Hidalgo, mientras que el 10 se refiere al caso de Guanajuato y la región Laja-Bajío.

Nos encontramos, por ende, con una bien estructurada composición del libro que le permite cubrir muy adecuadamente su propósito pedagógico, pues a partir de los conceptos generales de la disciplina desarrolla estudios específicos en una gran variedad de temas de la agenda regional de México. De inicio debemos recibir con beneplácito la aparición de un trabajo que cristaliza la valiosa experiencia que Mario Carrillo ha acumulado como destacado especialista en la denominada "ciencia regional" en las dos décadas en que escribió los artículos; con ello ha demostrado su gran vocación por la enseñanza.

Por cuestiones prácticas centraremos nuestros comentarios en los tres capítulos de la parte I, donde se presentan las cuestiones generales del proceso de diferenciación regional en México, esto es, en el ámbito nacional, así como los elementos conceptuales requeridos para su comprensión.

\section{Espacio y desarrollo regional}

El capítulo 1 es central para cumplir con el propósito de que el libro sirva como texto para los estudiantes de la especialidad, pues en él se presentan en forma sencilla y pedagógica algunas de las categorías centrales de la disciplina: el concepto de desarrollo, la naturaleza del espacio en los fenómenos regionales, así como el análisis de las regiones y la dialéctica de las desigualdades regionales, tema que es el hilo conductor que estructura todo el trabajo.

Evita de inicio conceptuar el desarrollo económico en términos simples y parte de la idea central de que constituye "un proceso mediante (y durante) el cual se mejora la calidad de vida de la sociedad; es decir como un mejoramiento en el bienestar social" (p. 10). Se habla más bien de un desarrollo social que incluye la cuestión económica, pero también la política y social. Después de explicar el concepto de proceso y la complejidad que adquiere al aplicarlo al desarrollo económico, concluye que "El simple hecho de que el ingreso real de 
una nación aumente durante un periodo largo, no implica que el bienestar económico de la sociedad mejore" (p. 16). En esta dirección cabe mencionar el libro $L$ a pérdida defelicidad en las democracias de mercado, del profesor emérito de la Universidad de Yale Robert Lane, donde valiéndose de series de información estadística para toda la segunda mitad del siglo Xx, demuestra que la felicidad individual ha disminuido significativamente, principalmente en Estados Unidos, pero también en otras democracias avanzadas (Lane, 2000).

Para vincular el desarrollo económico con el regional, Carrillo explica el concepto de región y sus tipos. Muy acertadamente expone de inicio que independientemente de lo abstracto que pueda ser el concepto de espacio, para el análisis territorial siempre tendrá que haber una "referencia en el espacio geográfico" (p. 20). Sea como fuere, para el análisis empírico de las regiones es preciso seleccionar ciertas variables que permitan medir su desarrollo. Explica y ejemplifica la cuestión de la medición con el índice de desarrollo humano utilizado por las Naciones Unidas. De acuerdo con este índice, los países cuyos valores son superiores a 0.799 tienen un desarrollo humano alto; los que están entre 0.500 y 0.799 tienen un desarrollo humano medio, y los inferiores a 0.500 , bajo. Para México se estima un IDH en 1998 de 0.785 , o sea que por 14 milésimas no somos desarrollados. Habrá que aclarar, por supuesto, que este tipo de taxonomías siempre presenta un grado de arbitrariedad considerable, por lo que hay que considerarlas con las reservas del caso.

Carrillo termina el capítulo con un cuadro sinóptico sobre la planeación del desarrollo urbano y regional, donde sintetiza algunas cuestiones conceptuales y se pregunta ¿Cómo ha operado la planeación urbana y regional en México? (p. 26). En fin, el capítulo se desarrolla en forma muy coherente y pedagógica, y sólo podría sugerirse que para futuras ediciones - ¡los libros de texto son otro proceso!- podría ser útil agregar los rasgos más notables del desarrollo económico mexicano, así como algunos de los planes urbanos y regionales elaborados por el gobierno federal.

\section{Desarrollo regional en México}

En el capítulo 2 se plantea reflexionar "sobre las perspectivas de un desarrollo más equilibrado regionalmente en el futuro en México”. Esta posibilidad, se agrega, dependerá de la efectividad del Estado 
mexicano para hacer los planes regionales necesarios para tal empresa, así como de que disponga del financiamiento adecuado y que la comunidad participe activamente (p. 34).

Cabría mencionar que el tema de las desigualdades regionales ha sido considerado en forma un tanto compulsiva por el gobierno federal como uno de los grandes problemas de la organización de las actividades económicas en el territorio nacional, pues todos los planes nacionales de desarrollo urbano incluyen la reducción de tales disparidades entre las metas centrales de las políticas en la materia. Dada la naturaleza de libro de texto del trabajo de Carrillo, convendría ir más allá del aforismo sobre la negatividad de las disparidades territoriales y cuestionar ¿por qué es deseable la mayor igualdad de las regiones del país?, y ¿hasta dónde es posible lograrla? Sólo cabe mencionar en esta oportunidad que no existe una relación estadística entre las desigualdades regionales y el nivel de desarrollo de los países. Japón, Francia e Inglaterra, por ejemplo, presentan grandes desigualdades regionales y son naciones desarrolladas.

Carrillo refiere que las disparidades en México se acentuaron entre 1940 y 1970 (p. 36), y adicionalmente, "que por lo menos hasta 1990, el desarrollo en México seguía siendo bastante desequilibrado regionalmente" (p. 36). En realidad, agregaríamos nosotros, existen varios estudios que concluyen que las desigualdades regionales disminuyeron en los años ochenta (Hernández, 1984: 161; Gómez y Cortés, 1987: 50; Osuna, 1990: 26). Sin embargo esta conclusión se obtiene utilizando a las entidades federativas como unidades de análisis, lo cual es inadecuado dada la existencia de varias conurbaciones entre estados de la República que hacen inapropiadas estas unidades. El ejemplo más notable es la Zona Metropolitana de la Ciudad de México, que al irse extendiendo desde los años cincuenta hacia el Estado de México, hace que el resto de las entidades se "acerque" al Distrito Federal, cuando en verdad esta entidad sólo constituye una fracción decreciente de toda la metrópoli.

En un estudio publicado en 2003 se concluye que de 1970 a 1999 ha habido cierta disminución de las desigualdades territoriales según grandes regiones y entidades de la República: la región centro-este, donde se encuentran el Distrito Federal y el Estado de México, redujo su participación en el PIB nacional de 43.0 a 41.7\%, mientras que la suma del Distrito Federal y el Estado de México pasó de 36.2 a 33.1\% (Garza, 2003a: 490). En el futuro, los estudios de las disparidades regionales deberán considerar a las ciudades como unidades de análi- 
sis, siendo que hasta ahora sólo el artículo anteriormente citado lo hace. En el ámbito interurbano, dicho trabajo concluye que en las manufacturas las desigualdades se reducen significativamente, pero en el sector terciario aumentan (Garza, 2003b). Estamos totalmente de acuerdo con la afirmación del autor respecto a que la promoción del desarrollo regional dejó de ser prioritaria para el gobierno federal (p. 39).

La parte más interesante del capítulo es un pormenorizado análisis del gasto público en el financiamiento del desarrollo en el país, sobre lo que se asegura que "ni el sistema de ingresos públicos o participaciones federales, ni el esquema de la inversión pública federal han promovido recientemente en México una redistribución de los recursos entre las entidades de la República” (p. 50). Aunque esto pueda ser correcto, convendría advertir a los estudiantes que los coeficientes de correlación de rangos (Spearman) aunque significativos, sus $\mathrm{R}^{2}$ son muy bajas y explican sólo una pequeña parte de la relación (por ejemplo, la R entre la inversión pública federal per capita y el PIB per capita de los estados es de 0.37 y la $\mathrm{R}^{2}$ de 0.13 ; p. 53). Igualmente sería muy útil explicar la fórmula que sigue la SHCP para distribuir las participaciones entre las entidades de la República, en la que pondera la población total, la captación de impuestos, y un indicador inverso al nivel de desarrollo. Por ejemplo, la participación del Distrito Federal y del Estado de México es de 25\% del total (p. 46), pero su PIB es alrededor de $35 \%$ del total nacional.

En fin, estoy seguro de que este capítulo será uno de los que más interesen a los estudiantes y futuros especialistas en la denominada "ciencia regional".

\section{Políticas de desarrollo regional en México}

El tercer capítulo tiene los siguientes objetivos principales: i) revisar más ampliamente la evolución de las desigualdades regionales en México; ii) discutir las políticas implementadas para promover un desarrollo territorial más equilibrado; iii) presentar los resultados de la evaluación de la política de parques industriales y la de empleo regional.

Más que ampliar el análisis de la dinámica de las desigualdades en el país, el primer inciso resume en un par de páginas lo expuesto en el capítulo 2. Al igual que en el capítulo anterior, sin embargo, sólo se 
describen las desigualdades hasta los años ochenta, por lo que sorprende que pese a que el artículo que constituye el capítulo se publicó en 1998, no se hayan agregado las estadísticas más recientes, así como una serie de estudios presentados en los últimos años.

$\mathrm{El}$ inciso referente a las políticas regionales enlista muy esquemáticamente dichas acciones siguiendo una cierta periodización de ellas. La sexta es la de las "Acciones para el reordenamiento territorial y mejoramiento solidario de la calidad de vida de la población, 19891994", pero como en este periodo los salarios contractuales reales de la clase trabajadora se redujeron $-10.4 \%$ y los mínimos $-29.1 \%$, más bien resultó en la etapa de "Acciones de empeoramiento unilateral de la población”. En un libro recientemente aparecido, a la última etapa de las políticas territoriales en México se le denomina: "Abdicación de las funciones de planeación del espacio nacional, 1988-2003" (Garza, 2003a: 101), por lo que en ella se incluyen el Plan Puebla Panamá y el Programa Nacional de Desarrollo Urbano y Ordenación del Territorio, 2001-2006, actualmente vigentes.

El programa de parques y ciudades industriales se describe en tres páginas, por lo que sólo se presenta una visión muy general de la cuestión, en parte por el carácter sintético que debe tener un libro de texto. En realidad, únicamente se sintetiza un libro de Ismael Aguilar Barajas, por lo que sólo se vislumbran las características de una de las más sistemáticas e importantes acciones territoriales del Estado mexicano.

El capítulo 3 termina con una síntesis de la interesante evaluación de los Programas Regionales de Empleo (PRE) que fueron desarrollados en los años ochenta y sobre los cuales Carrillo compiló un libro que apareció en 1986. Los PRE fueron evaluados mediante ocho instrumentos diferentes en su etapa de diseño, realización y operación, utilizando información recolectada mediante alrededor de 1500 entrevistas cuyos resultados se procesaron utilizando la técnica de componentes principales del análisis factorial. Se concluyó que los programas más efectivos en relación con el impacto en el empleo fueron el del servicio social de pasantes; el de colonias populares según ingreso; el de proyectos productivos para el bienestar y los proyectos de infraestructura básica para propósitos del desarrollo regional (p. 80).

En cuanto a las conclusiones del capítulo, concordamos completamente con la visión del autor sobre el abandono de la planeación regional desde mediados de los ochenta y la tendencia a transferir esa función a los gobiernos estatales y municipales. El futuro de la 
planeación territorial dependerá, concluye Carrillo, "de los esfuerzos autónomos que se hagan a nivel local y regional, y de las relaciones que existan entre los estados y la federación, lo que a su vez dependerá de la recomposición de fuerzas políticas" (p. 84). Cabría concluir, por nuestra parte, que las perspectivas a ese respecto no son muy halagüeñas, pues desde entonces tanto la federación como los estados y municipios evidencian cierta trivialización de la gestión pública, ya que el gasto en publicidad está muy por encima de la inversión en planeación y en la optimización de las acciones de gobierno. Mientras tanto, cada año México va perdiendo aceleradamente competitividad respecto al resto del mundo.

En las consideraciones finales del capítulo 10, último del libro, Carrillo ve con optimismo las iniciativas del estado de Guanajuato de establecer en 1996 un marco jurídico para crear un sistema de programación regional dirigido por un Consejo de planeación del desarrollo del estado de Guanajuato, conformado por miembros de todos los sectores sociales. La experiencia de un organismo similar en Nuevo León —el Consejo Consultivo de Desarrollo Urbano- ha sido poco fructífera, y en algunos casos los organismos de este tipo constituyen mecanismos políticos que con la participación de la ciudadanía encubren a los verdaderos centros de poder que toman las principales decisiones de la acción pública.

No sabemos cuál sería el balance de la experiencia de Guanajuato, pero existe cierta evidencia de que se trató de acciones voluntaristas de tipo empresarial con muy limitados resultados. Esto se refleja en la participación del PIB de Guanajuato en el total nacional, que se reduce de $3.38 \%$ en 1996 a $3.26 \%$ en 2000 (www.inegi.gob.mx; cuentas nacionales por entidad federativa). Así, la promoción del desarrollo del gobierno estatal en ese sexenio no logró que Guanajuato creciera ni siquiera al nivel de la media nacional.

Quizás sea prematuro realizar una profunda evaluación del impacto de los gobiernos y las políticas neoliberales sobre las desigualdades y perspectivas de la planeación territorial en México, pero indudablemente Mario Carrillo ha puesto con este libro su granito de arena para renovar el debate nacional sobre la cuestión del desarrollo regional y las grandes disparidades en la distribución territorial de la población y las actividades económicas en México. 


\section{Bibliografía}

Garza, Gustavo (2003a), La urbanización de M éxico en el siglo Xx, México, El Colegio de México.

(2003b), "The Dialectics of Urban and Regional Disparities in Méxi-

co", en Kevin J. Middlebrook y Eduardo Zepeda (eds.), Confronting Development. A ssessing M exico's Economic and Social Policy Challenges, Estados Unidos, Stanford University Press.

Gómez, Pablo y Armando Cortés (1987), Experiencia histórica y promoción del desarrollo regional en M éxico, México, Nacional Financiera.

Hernández Laos, Enrique (1984), "La desigualdad regional en México (19001980)", en Rolando Cordera y Carlos Tello (coords.), La desigualdad en M éxico, México, Siglo Xxi Editores.

Lane, Robert E. (2000), TheL oss of H appiness in M arket Democracies, New HavenLondres, Yale University Press.

OECD (1997), Regional Development and Structural Policy in M éxico, París, Organisation for Economic Cooperation and Development.

Osuna, Germán (1990), "Dinámica de la desigualdad regional en México, 1970-1980", Estu dios Demográficos y U rban os, vol. 5, núm. 1 (13), pp. 5-35.

Ramírez, Delfina (1986), "Las desigualdades interregionales en México, 19701980", Estudios Demográficos y U rbanos, vol. 1, núm. 3(3), pp. 351-376. 